\title{
The self-interaction of native TDP-43 C terminus inhibits its degradation and contributes to early proteinopathies
}

\author{
I.-Fan Wang ${ }^{1,2}$, Hsiang-Yu Chang1,2,3, Shin-Chen Hou ${ }^{2,3}$, Gunn-Guang Liou ${ }^{4}$, Tzong-Der Way ${ }^{3}$ \\ \& C.-K. James Shen ${ }^{1}$
}

The degraded, misfolded C terminus of TAR DNA-binding protein- 43 is associated with a wide spectrum of neurodegenerative diseases, particularly frontotemporal lobar degeneration with ubiquitin-positive inclusions and amyotrophic lateral sclerosis. However, the precise mechanism of pathological cleavage of the TAR DNA-binding protein-43 remains unknown. Here we show that the TAR DNA-binding protein-43 C-terminal protein physically interacts with itself or with the cellular-folded yeast prion domain of Sup35 forming dynamic aggregates. This prion-like nature governs known cellular functions of the TAR DNA-binding protein-43, including subcellular localisation and exon skipping of the cystic fibrosis transmembrane conductance regulator. Significantly, mutants with a failure to engage in prion-like interactions are processed into an $\sim 24-k D a$ C-terminal fragment of the TAR DNA-binding protein- 43 . The estimated cleavage site of degraded TAR DNA-binding protein-43 fragments corresponds to the pathological cleavage site identified in patients with the TAR DNA-binding protein-43 proteinopathies. Consistently, epigallocatechin gallate constrains prion-like interactions, attenuating pathological-like degradation. Thus, the native folding of TAR DNAbinding protein- $43 \mathrm{C}$ terminus acts as a guardian of pathogenesis, which is directly associated with loss-of-function.

\footnotetext{
${ }^{1}$ Institute of Molecular Biology, Academia Sinica, Taipei 115, Taiwan. ${ }^{2}$ Garage Brain Science, Taichung 413, Taiwan. ${ }^{3}$ Department of Biological Science and Technology, College of Life Sciences, China Medical University, Taichung 404, Taiwan. ${ }^{4}$ Institute of Molecular and Genomic Medicine, National Health Research Institutes, Miaoli 350, Taiwan. Correspondence and requests for materials should be addressed to I.F.W. (email: mirna125@gmail.com).
} 
$\mathrm{T}$ he aggregation of misfolded, phosphorylated, ubiquitinated C-terminal fragments of TAR DNA-binding protein 43 (TDP-43) is a common characteristic in frontotemporal lobar degeneration with ubiquitin-positive inclusions and amyotrophic lateral sclerosis (ALS) ${ }^{1-8}$. Among mammals, zebrafish and flies, the protein sequence, biochemical properties and biological functions of TDP-43 are well conserved. In the nucleus, TDP-43 forms visible 50-250-nm granules that serve as a scaffold to link other functionally related sub-compartments, and participates in transcriptional repression as well as alternative splicing ${ }^{9-12}$. TDP-43 specifically binds to the UG repeat sequences in RNA, altering pre-mRNA splicing of cystic fibrosis transmembrane conductance regulator (CFTR) and may associate with long non-coding RNA to regulate nuclear speckle or paraspeckle ${ }^{11,13}$. TDP-43 mutants lacking a C-terminal glycine-rich region fail to excise exon 9 in the CFTR gene ${ }^{9}$, but how TDP-43 C terminus governs exon 9 skipping of CFTR, as well as its role in pathogenesis of TDP-43 proteinopathies, is still unknown. Given that the TDP-43 C terminus binds directly to heterogeneous nuclear ribonucleoprotein (hnRNP) A1 and hnRNP A2/B1 in pull-down assays ${ }^{14}$, the loss of association between TDP-43 and the hnRNPs has been proposed as a possible mechanism behind TDP-43 proteinopathies, causing aberrant pre-mRNA splicing or mislocalisation of TDP-43. However, this hypothesis does not adequately account for pathological degradation of TDP-43 protein itself, which is a major feature of TDP-43 proteinopathies in the early stage ${ }^{1}$.

Recently, we have reported a database search of homologues of the mTDP- $43 \mathrm{C}$ terminus across multiple species and found that the mTDP-43 C-terminal domain (amino acids (a.a.) 274-414) shares $24.2 \%$ sequence identity with the $\mathrm{N}$-terminal yeast prion domain of Sup35 (a.a. 1-125; SupN) ${ }^{15}$. This analysis led us to propose that the TDP- $43 \mathrm{C}$ terminus might have prion-related properties. On the basis of the atypical Q/N-rich domain of TDP-43 involved in the self-assembly of misfolded C-terminal fragments and the sequestration of TDP-43 into polyglutamine aggregates, TDP-43 has been further proposed to act as a prion ${ }^{16}$. However, in contrast to most known prions, misfolded pathological fragments of TDP- 43 or fulllength TDP-43 (TDP-43-FL) aggregates in vitro do not react with the amyloid-specific dye Congo red ${ }^{1,17}$, indicating that the TDP-43 $\mathrm{Q} / \mathrm{N}$-rich domain may not be a prionogenic domain.

We were thus interested in understanding whether the sequence similarity in Q/N residues between the TDP-43 C-terminal and known prion domains is critical to the cellular functions of TDP$43 \mathrm{C}$ terminus, rather than misfolded-related. However, to date, no cellular roles have been clearly elucidated for a prion domain. To gain insight into its cellular role, we developed assays to explore the native functions of TDP-43 C terminus in cells. We demonstrated a novel intrinsic property of the TDP-43 C terminus for cellular functions, including pre-mRNA splicing, nuclear granular assembly and protein stability, which revise the concept of the prion domain.

\section{Results}

Functional aggregation of TDP-43 via GQN-rich C terminus. On the basis of structure and intensity, two groups of TDP-43 clusters appeared in the nucleus: visible granules of $50-250 \mathrm{~nm}$ in diameter (arrow, Fig. 1a) and irregular puncta (arrowhead, Fig. 1a), as shown by endogenous TDP-43 and green fluorescent protein (GFP)-tagged, FL mTDP-43 (TDP-43-FL; Fig. 1a). The dynamics of TDP-43 clusters in living cells were further visualised by time-lapse microscopy. In GFP-TDP-43-FL-transfected cells, the small irregular puncta were rapidly burst, reorganised, or disassembled, and were restricted to a local area (Fig. 1b). High magnifications of irregular puncta of GFP-TDP-43-FL are shown in Fig. 1b (arrowheads). Individual GFP-TDP-43 small puncta histories suggest that they have similar motility characteristics. In contrast, most of the visual granules were more stable and travelled within a limited localisation. A few granules were stationary (white arrowheads, Fig. 1c). Also observed, but rare, were fission and fusion events (yellow arrowheads and red arrowheads, respectively, Fig. 1c). Occasionally, two independent granules of GFP-TDP-43-FL from two different areas collided and then separated (blue arrowheads), possibly exchanging material between granules (Fig. 1c). With gentle $0.5 \%$ Triton $\mathrm{X}-100$ pre-extraction of cells to remove diffuse nucleoplasmic TDP-43 proteins, TDP-43 clusters in the nuclei exhibit clear shapes by immunostaining (Fig. 1d ${ }^{18}$. An example of a nucleus is shown in Fig. 1d. No signal was detected in cells without permeabilisation (Fig. 1d). Western blot further confirmed that a fraction of mTDP43-FL proteins was retained in the detergent-insoluble fraction (Fig. 1e). These results suggest that these insoluble supermolecules of TDP- 43 protein behave as highly dynamic functional aggregates in the nucleus.

Overexpressing GFP-tagged GQN-rich C terminus of TDP-43 was evenly distributed in the nucleus and cytosol (Fig. 1f). However, in $~ 15 \%$ of GFP-tagged GQN-rich C terminus transfectants, we found significantly altered cell morphology, and the GQN-rich C-terminal proteins appeared in a filamentous pattern in the cytosol (Fig. 1f). Furthermore, ultrastructural analysis of GFP-tagged TDP43 C-terminal proteins revealed tightly packed 6- to 8-nm diameter filaments in the cytosol (Fig. $1 \mathrm{~g}$ ). We thus termed the region as prion-like domain (PLD) ${ }^{19}$. When PLD was expressed in primary neurons, they formed foci in the dendrites (Fig. 1h). These results indicate that an atypical Q/N-rich domain of TDP-43 is capable of spontaneously forming a filamentous structure and retains TDP-43 proteins in the insoluble fraction in mammalian cells in the same way as other aggregation-prone domains (Fig. $1 \mathrm{f}-\mathrm{h}$ ). To validate whether the aggregation propensity of TDP-43 PLD contributes to forming functional TDP-43 aggregates in normal cells, we constructed a TDP-43 C-terminal deletion mutant, TDP-43-PLD $\Delta$. In the absence of the PLD, TDP-43-PLD $\Delta$ diffused in the nucleus (Fig. 1i). It appears that, in addition to forming misfolding aggregates, the $\mathrm{Q} / \mathrm{N}$-rich domain is essential for the assembly of reversal functional TDP-43 aggregates in cells.

\section{Functional substitutions of TDP-43 C terminus by prion domain.} To clarify the intriguing sequence similarity in $\mathrm{Q} / \mathrm{N}$ residues between the TDP-43 GQN-rich C-terminal and yeast prion domain of Sup35 (SupN), we replaced the PLD of GFP-tagged mTDP-43 with yeast prion domains (TDPSupN; Fig. 2a). As the sequence homology or the polyQ/ $\mathrm{N}$ context may affect the conformation in cells, an additional replacement by the N-rich yeast prion domain, UreN (a.a. 1-84) was also generated. UreN shares only $12 \%$ similarity with the PLD. The distribution of the $\mathrm{Q}$ and $\mathrm{N}$ residues of these three $\mathrm{Q} / \mathrm{N}$-rich domains was illustrated in Fig. 2b. Interestingly, chimeric TDPSupN proteins restored two groups of clustering aggregates: visible granules of $50-250 \mathrm{~nm}$ in diameter (arrow, Fig. 2c) and irregular puncta (arrowhead, Fig. 2c) in the nuclei, as shown by the GFP-tagged, mTDP-43-FL. In contrast, mTDPUreN failed to recapitulate TDP-43-FL localisation, although mTDPUreN formed large foci in the cytosol (Fig. 2c). Live-cell imaging showed that the clustered mTDPSupN proteins had identical behaviour to mTDP-43-FL. Fluorescence recovery after photobleaching analysis of GFP-mTDP-43-FL and GFP-mTDPSupN movement showed that fluorescence recovery of these proteins was rapid, but significantly slower than that of mTDP-43-PLD $\Delta$ (Fig. 2d). The half-recovery time was calculated to be $8.23,7.28$ and $1.34 \mathrm{~s}$, for GFP-mTDP43-FL, GFP-mTDPSupN and GFP-mTDP-43-PLD $\Delta$, respectively. The recording images obtained from two bleached areas of a single GFP-mTDPSupN-expressing cell showed fluorescence recovery (Fig. 2e). We noticed that the fluorescence recovery in visible granules was faster than that in irregular puncta, which may imply differential kinetics in the redistribution of protein into the two types of functional aggregates (Supplementary Movie 1). Significantly, 
a

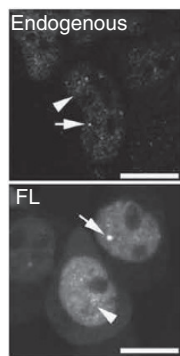

b

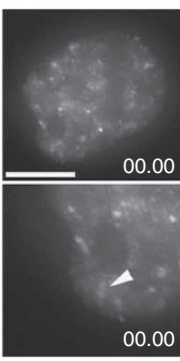

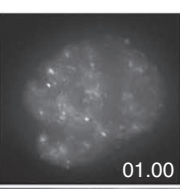
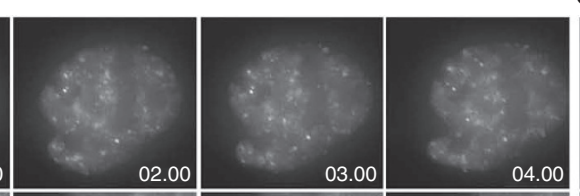

d
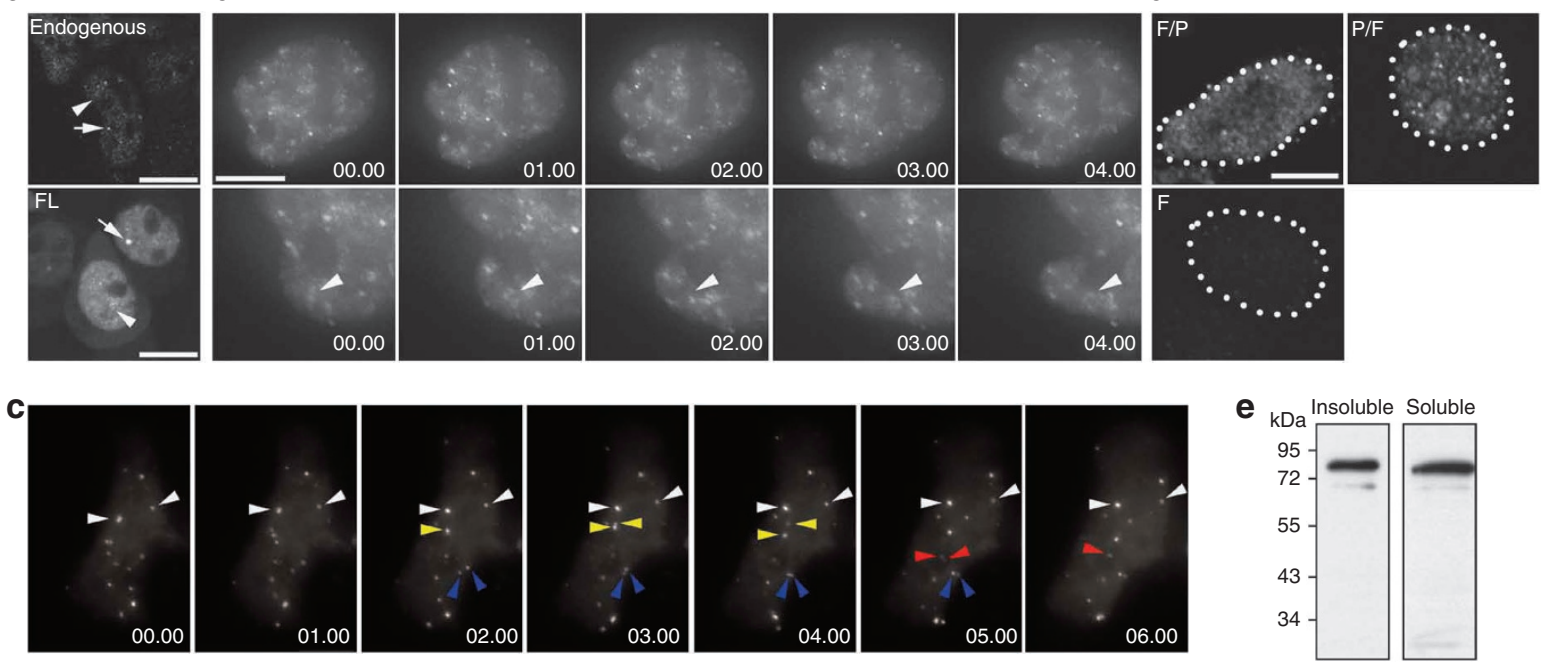

f

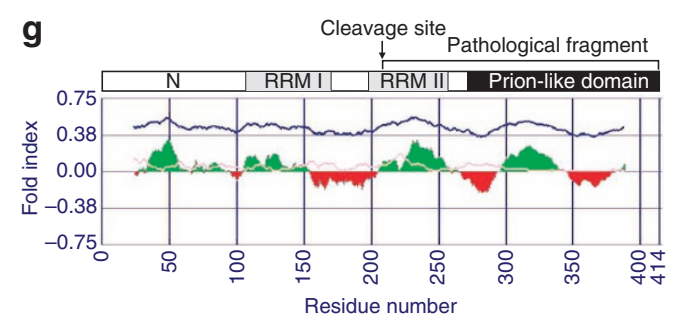

h

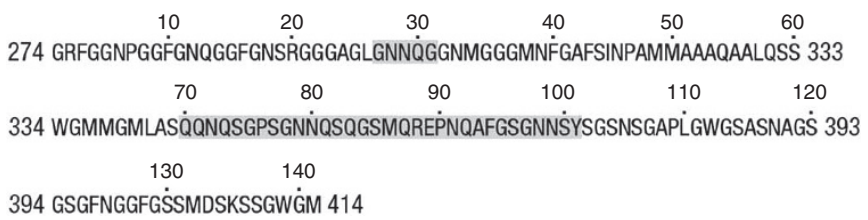
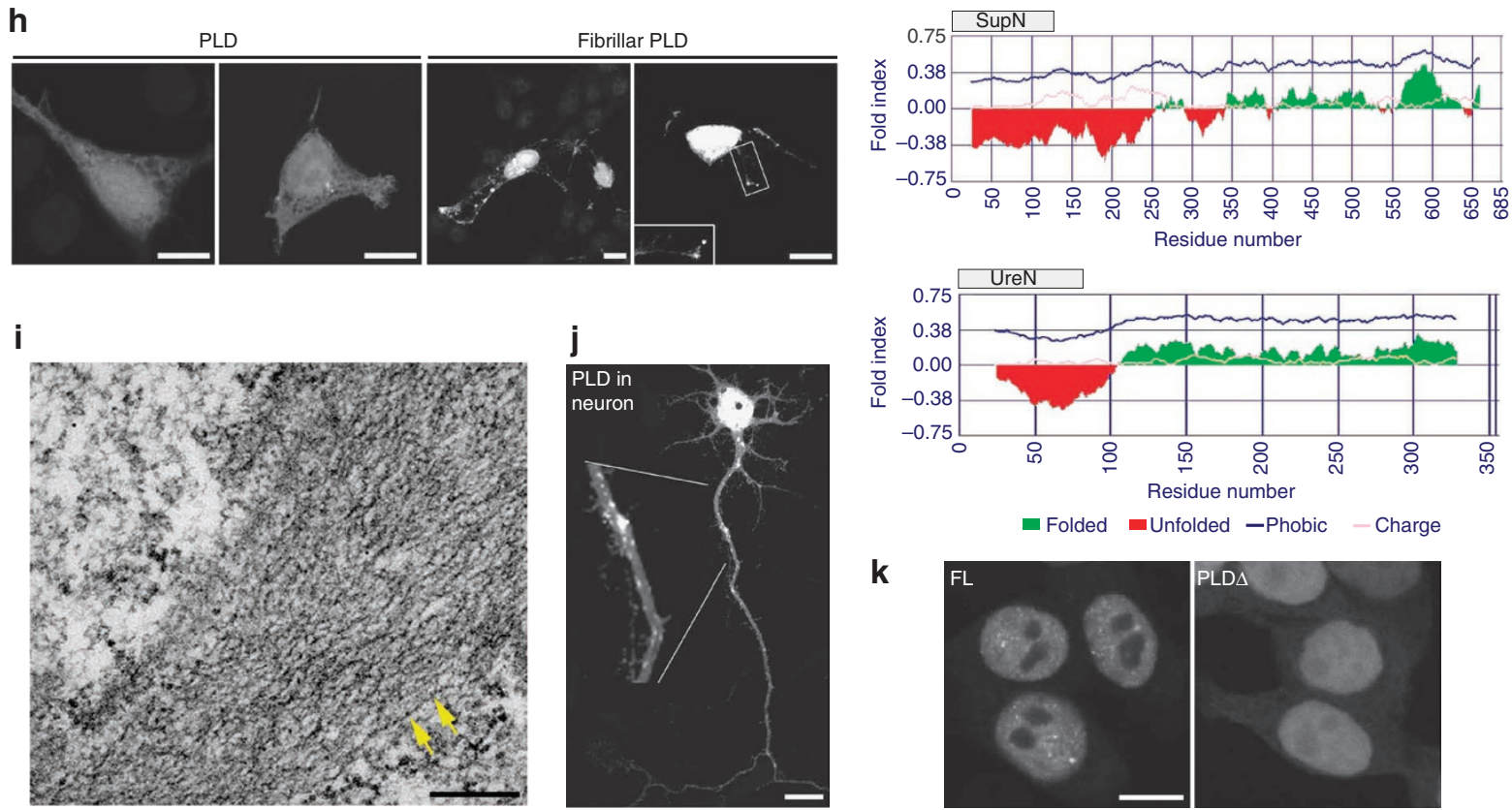

$\mathbf{k}$
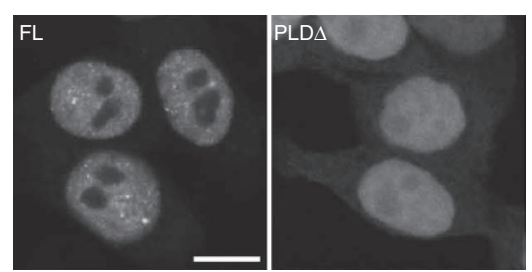

Figure 1 | Dynamics of detergent-insoluble TDP-43 aggregates in normal cells. (a) GFP-mTDP-43-FL forms dispersed visible granules (arrow) and irregularly shaped puncta (arrowhead) in the cell nucleus. Scale bars represent $10 \mu \mathrm{m}$. (b) Visualisation of supramolecular GFP-mTDP-43-FL proteins by time-lapse microscopy, images of GFP-mTDP-43-FL-expressing cells were taken every 1 for $30 \mathrm{~min}$. Arrowheads in high-magnification images, upper panels, show the dynamics of irregularly shaped puncta. Scale bars represent $10 \mu \mathrm{m}$. (c) Tracking of visible granules of GFP-mTDP-43-FL. White arrowheads, stationary granules; yellow arrowheads, fission; red arrowheads, fusion; blue arrowheads, collision and separation of two distinct granules. (d) Pre-extraction of nucleoplasmic proteins revealed clear TDP-43 aggregates in the 293T cells. F/P, fixation/permeabilisation, transfectants were fixed with 3.75\% paraformaldehyde and permeabilised with $0.5 \%$ Triton X-100. Pre-extraction protocol (P/F), transfectants were extracted with $0.5 \%$ Triton X-100, and then fixed with $3.75 \%$ paraformaldehyde. Negative immunostaining control, cells without permeabilisation (F). Fixed 293T cells were immunostained with antibodies against TDP-43. (e) Validation of the solubility of GFP-mTDP-43-FL by western blot with anti-GFP antibody. (f) Peptide sequence of the GQN-rich C terminus of mTDP-43 (PLD, prion-like domain). The two Q/N-rich regions are marked in gray. (g) Foldlndex prediction of unstructured regions in mTDP-43, yeast prion Sup35 and Ure2p.

(h) The mTDP-43-PLD construct was transfected into 293 T cells. Note that $15 \%$ of mTDP-43-PLD transfectants with fibrillar mTDP-43-PLD in the cytosol had neurite-like shapes. (i) Ultrastructural analysis of GFP-tagged mTDP-43-PLD is shown at high magnification. Arrows, filamentous structure. Scale bar represents $0.2 \mu \mathrm{m}$. (j) In primary culture neurons, GFP-mTDP-43-PLD aggregated into small puncta. Inset magnified image depicting neurite accumulation. Scale bars represent $10 \mu \mathrm{m}$. (k) Even nuclear distribution of mTDP-43-PLD $\Delta$ transfected into $293 \mathrm{~T}$ cells. Scale bar represent $10 \mu \mathrm{m}$. 


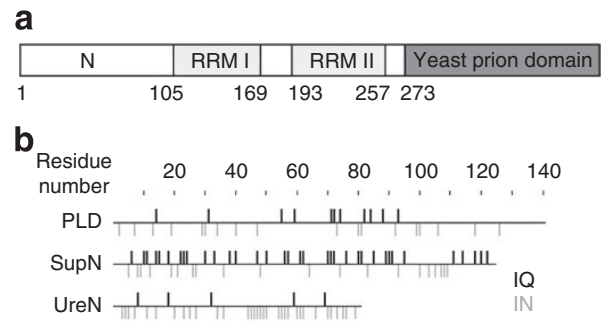

d

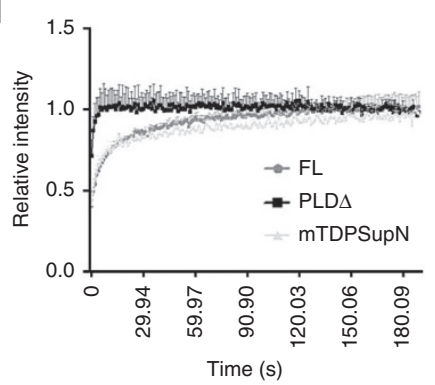

f

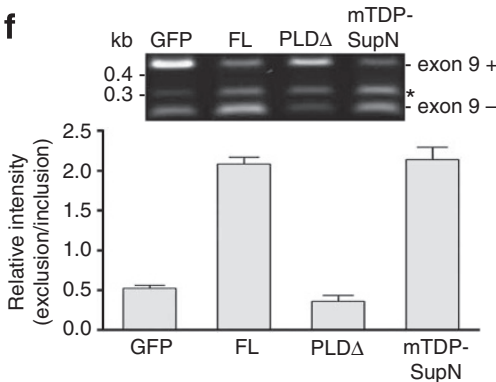

\section{C}

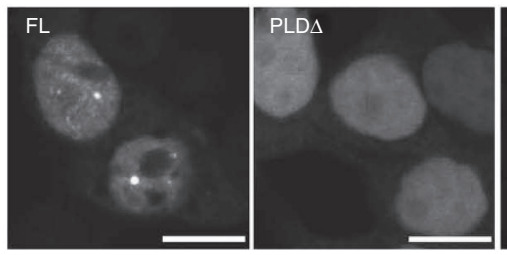

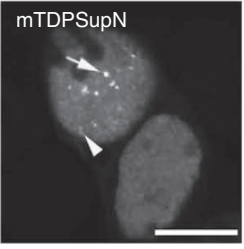

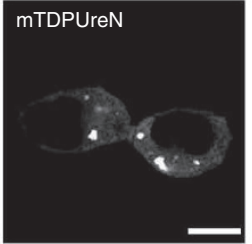

.

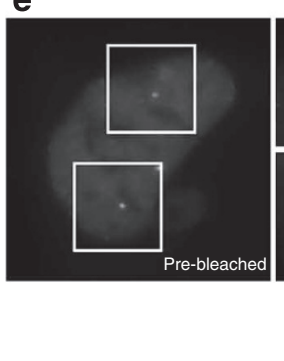

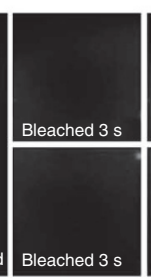
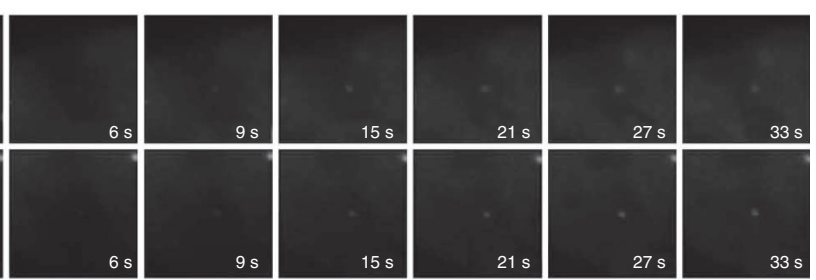

g

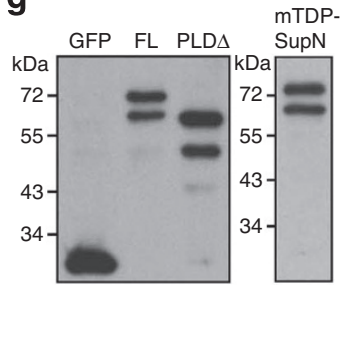

h

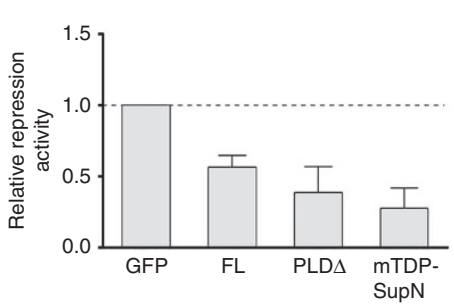

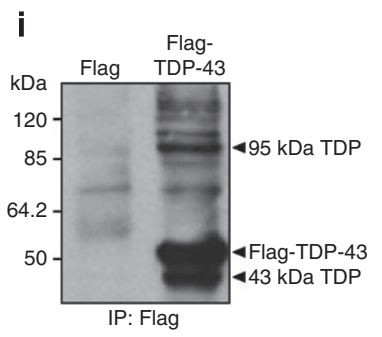
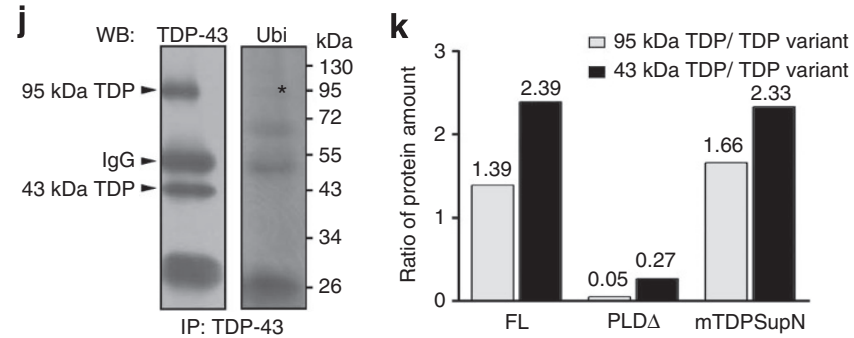

Figure 2 | Functional substitutions of GQN-rich C terminus of $\mathbf{m T D P}-\mathbf{4 3}$ by a yeast prion domain SupN. (a) Scheme of GFP-tagged chimerical mTDP-yeast prion domain fusion construct. (b) Illustration of Q/N distribution in the PLD, yeast prion domain Sup35 (1-125; SupN) and UreN (1-84). (c) Localisation of GFP-tagged mTDPSupN and mTDPUreN. The construct mTDPSupN formed dispersed visible granules (arrow) and irregularly shaped puncta (arrowhead) in the cell nucleus. The mTDPUreN formed large foci in cytosol. Scale bars represent $10 \mu \mathrm{m}$. (d) Fluorescence recovery after photobleaching analysis of GFP-TDP-43-FL, GFP-TDPSupN and GFP-TDP-43-PLD $\Delta$ movement by time-lapse microscopy. (e) Selected images of TDPSupN expressing cells demonstrate fluorescence recovery of GFP-tagged TDP-43 proteins. (f) Further examination of the alternative splicing ability of GFP-tagged mTDPSupN by in-vivo splicing assay. Exon-9 inclusion (+) and exon-9 exclusion (-) bands are indicated. ${ }^{*}$, aberrant splicing product. (g) Immunoblot analysis of the level of GFP-tagged transfectants used in the examination of exon 9 skipping of CFTR. (h) Transcription activity assay. (i) An examination of TDP-43 self-interaction in vivo by cross-immunoprecipitation (IP). The protein lysate harvested from Flag- or Flag-TDP-43expressing 293 T cells was used for immunoprecipitation with anti-Flag antibody and was further examined by immunoblotting with anti-TDP antibody. (j) An examination of $95 \mathrm{kDa}$ TDP-43 by cross-IP. The copurification of TDP-43 with associated proteins was performed by cross-IP analysis with anti-TDP-43 antibody from the lysate of 293T cells and was further examined by immunoblotting with anti-TDP antibody or anti-ubiquitin antibody. *, ubiquitinated $95 \mathrm{kDa}$ TDP-43. (k) Coprecipitation efficiency of mTDP-43 variants with $95 \mathrm{kDa}$ TDP and $43 \mathrm{kDa}$ TDP. The copurification of TDP-43 with associated proteins was performed by cross-IP analysis with anti-GFP antibody from the lysate of overexpressing GFP-tagged mTDP-43 variants FL, PLD $\Delta$ or TDPSupN. The immunoprecipitates were further examined by immunoblotting with an anti-TDP antibody. SupN complemented PLD in interaction with endogenous TDP. All data are presented as means with s.d. $(n=3)$.

in vivo splicing assays revealed that mTDPSupN effectively promoted exon 9 skipping of CFTR in the same way as TDP-43-FL (Fig. 2f-g). Cotransfection with the $\alpha$-globin promoter and $\operatorname{mTDPSupN}$ in $293 \mathrm{~T}$ cells did not alter mTDP-43 transcription repression (Fig. 2h). As expected, the $\mathrm{C}$ terminus of TDP-43 was not involved in TDPmediated transcriptional repression ${ }^{12}$. In summary, mTDPSupN localised to the nucleus, where it effectively promoted exon 9 skipping in the same manner as endogenous TDP-43.

As GFP-TDP-43-FL and GFP-TDPSupN proteins dynamically clustered in the nucleus, we examined whether self-interaction of TDP-43 occur via PLD. Using coimmunoprecipitation with FLAG antibodies, we found that endogenous 43 and $95 \mathrm{kDa}$ TDP proteins 
coprecipitated with FLAG-tagged TDP-43, suggesting TDP-43 self-interaction (Fig. 2i). A $95-k D a$ TDP-43 protein was likely constituted by a $43-\mathrm{kDa}$ TDP-43 and a $52-\mathrm{kDa}$ ubiquitinated TDP-43 (Fig. 2j). This self-interaction can be recapitulated by the GFP-tagged TDP-43-FL and mTDPSupN (Fig. 2k). Notably, the coprecipitation efficiency of endogenous TDP-43 with mTDPSupN was similar to that with TDP-43-FL, and more effective than with TDP-43-PLD $\Delta$ (Fig. 2k). These results suggested that the cellular role of the $\mathrm{Q} / \mathrm{N}$ rich domain is to promote protein self- or cross-interactions and act as a protein-protein interaction domain in the cell.

Comparisons among the cellular and misfolded properties of the known $\mathrm{Q} / \mathrm{N}$-rich domain are shown in Table $1^{20-31}$. The cellularfolded $\mathrm{Q} / \mathrm{N}$-rich domain shared key properties characteristic of the misfolded proteins, including insolubility and a tendency to form aggregates via intrinsic element- and self-interactions. However, the interaction of these prion-like supramolecules was dynamic in the cell in response to stimuli. We hypothesised that these interactions occur because of the structural plasticity of the aggregation-prone domain, which allows temporal folding of parallel $\beta$-sheets in the cell. We thus described this interaction of cellular-folded Q/N-rich domain as 'prion-like interaction'. Although natively folded Sup35N and TDP-43 C terminus adopt substitutable structure in cells, only Sup35N aggregate into an in-register $\beta$-sheet amyloid structure when misfolded, suggesting that a protein that has prion-like properties does not have to be a prion.

A single proline substitution induced pathological degradation. Substitution of the structural breaking amino acid proline has been demonstrated to destroy $\beta$-sheets ${ }^{32}$. The simplified model is illustrated in Fig. 3a. As glycine-rich region localises at adjacent, the first Q/N-rich region, two glycine residues, G296 and G300, were selected to examine the effect of glycine on the prion-like nature of TDP-43 C terminus. We substituted proline at amino acids G296, G300, Q303 or Q343 of TDP-43 PLD (Fig. 3b). Notably, only TDP-43 Q303P proteins were partially degraded into $\sim 24 \mathrm{kDa}$ pathological fragments (asterisk, Fig. 3b). An in-vivo splicing assay showed that TDP-43 Q303P attenuated exon 9 skipping efficiency by $23.5 \%$ (Fig. 3c). Four days post-transfection with Q303P, a portion of the transfected cells showed cytoplasmic accumulation of TDP-43 Q303P fusion proteins (arrow, Fig. 3d) and formed cytosolic aggregates (arrowhead, Fig. 3d). The ratio of cells with cytoplasmic accumulated fusion proteins in TDP-43-FL- and TDP-43Q303P-expressing transfectants is shown in Fig. 3e. Indeed, a subset of the TDP-43-Q303P-expressing cells had a lower fluorescence half-recovery time, which was similar to that of TDP-43 PLD $\Delta$ (Fig. 3f). A lower viability of TDP-43-Q303P-expressing cells was further confirmed by the MTT (3-(4,5-(dimethylthiazol-2-yl)-2, 5-diphenyltetrazolium bromide) assay (Fig. $3 g$ ).

Substitution of proline at amino acid 25 of Sup35 has been suggested to abolish the propagation and infectivity of all three [PSI] variants $^{33}$. To confirm that impairing the cellular properties of the 'prion domain' leads to pathological degradation of TDP-43 proteins, we generated a mTDPSupN mutant, GFP-mTDPSupN*, which contained a G25P substitution in SupN, and assessed its localisation and ability to skip exon 9 of CFTR. As expected, immunoblot analysis showed that the majority of GFP-mTDPSupN* proteins shifted to the soluble fraction and were degraded into $\sim 48 \mathrm{kDa}$ fragments (Fig. 3h). The estimated cleavage site of the degraded mTDPSupN* fragment corresponded to the cleavage site of an $\sim 24 \mathrm{kDa}$ fragment that has been detected in patients with TDP-43 proteinopathies ${ }^{1}$. Tubulin and actin immunoblot were used as controls for non-specific protein degradation (Fig. 3h). The degraded mTDPSupN* fragment did not translocate into the insoluble fraction, possibly because a G25P substitution in SupN* completely abolished the intrinsic propensity of mTDPSupN* to aggregate. GFP-mTDPSupN ${ }^{\star}$ was evenly distributed in the nucleus and cytosol (Fig. 3i), and failed Table 1 | Properties of cellular folding and misfolding of
Q/N-rich domain.

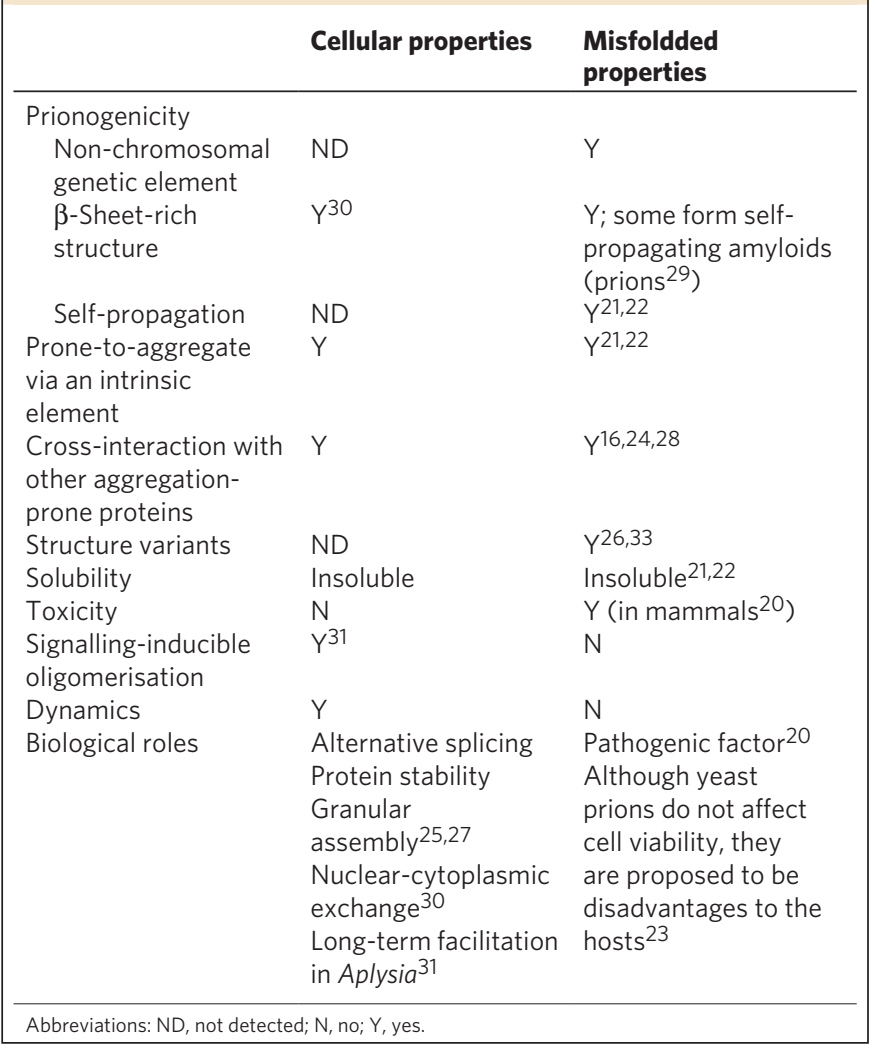

to promote alternative splicing activity that confirmed loss of functional PLD of TDP-43 (Fig. 3j).

As most of the ALS-inherited mutations are clustered in the $\mathrm{C}$ terminus of TDP- $43^{3}$, we further examined whether these mutations attenuated the prion-like activity of TDP-43. We generated two disease mutants by introducing point mutations at G348C and R361S, and analysed the prion-like activity of these mutants by measuring the CFTR exon 9 skipping efficiency and assembly of TDP-43 functional aggregates in the nucleus. Compared with TDP-43-FL, G348C and R361S mutants attenuated exon 9 skipping efficiency by 12.5 and $28.9 \%$, respectively, whereas G296P, a disease-unrelated mutant, did not (Fig. 3k). Unfortunately, immunoblot analysis of the lysate from the same preparation of alternative splicing assay did not show an increase in degraded fragments in either the soluble or insoluble fractions in G348C- and R361S-expressing cells at $48 \mathrm{~h}$ post-transfection (Fig. 31); meanwhile, both TDP-43 G348C and TDP-43 R361S proteins were able to assemble nuclear aggregates normally and did not increase cytoplasmic redistribution in 293T cells (Fig. 3m). We do not know why these two ALS-inherited mutation proteins could not recapitulate their pathological characters in a cell-based overexpression system, but we noticed that the slight effect of these two mutations on exon-skipping defect correlated with slightly increased protein degradation shown in previous studies $^{3}$. We deduced that this slightly decreased efficiency results from incomplete disruption of the prion-like folding, and this result may thus support our hypothesis that impairment of prion-like activity occurs before protein degradation in mutation-linked ALS patients.

Attenuation of TDP-43 degradation by EGCG. If the disruption of prion-like interactions of the Sup35 or TDP-43 C terminus leads to a shift into the soluble fraction and protein degradation (Fig. 3), 
a

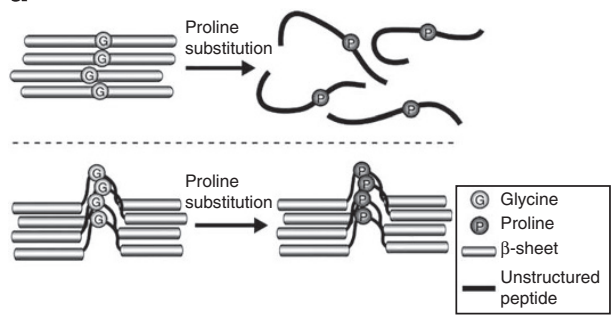

b

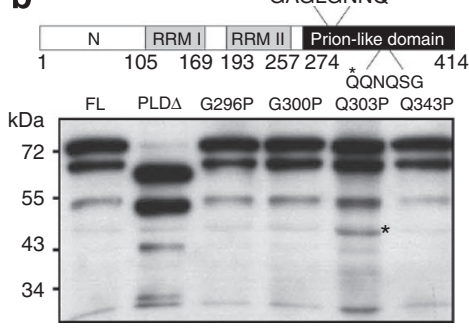

C

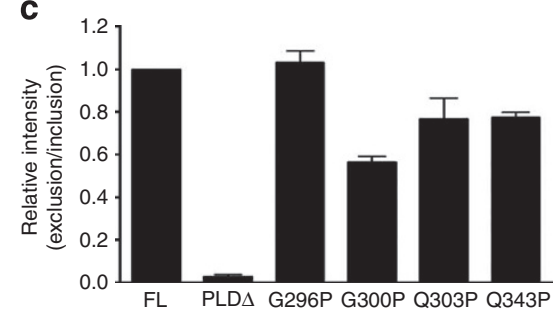

d
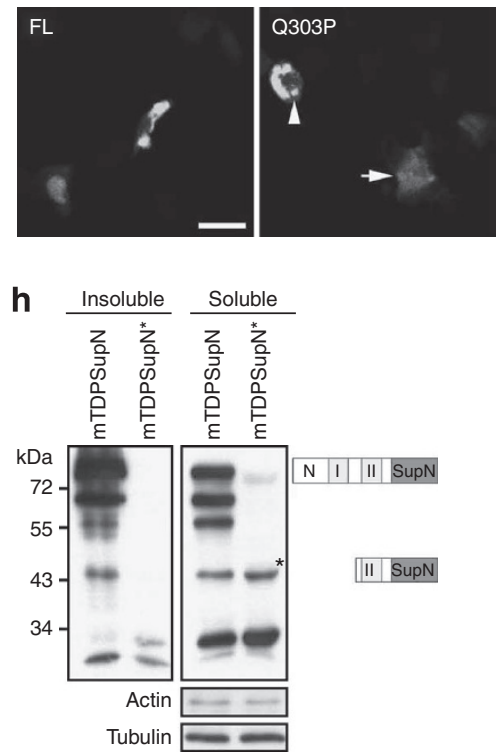

e

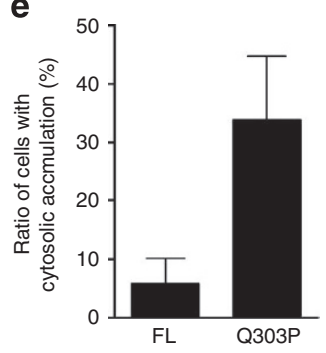

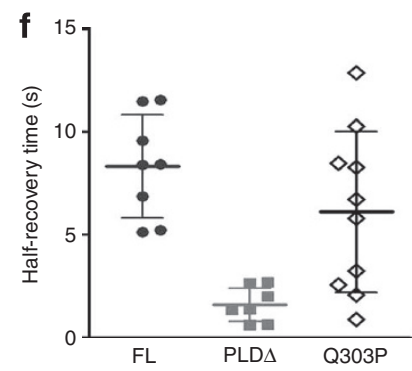

g

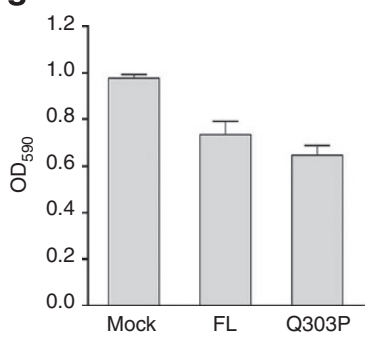

i

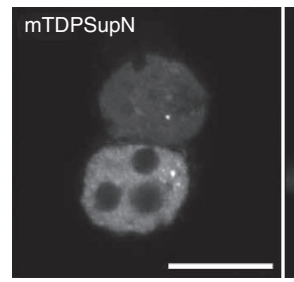

j
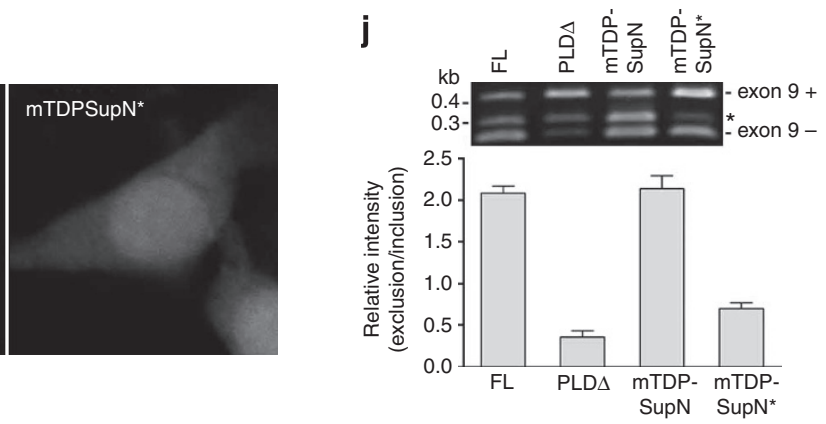

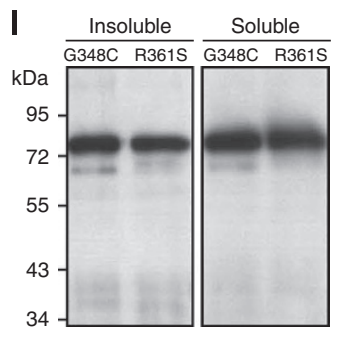

m

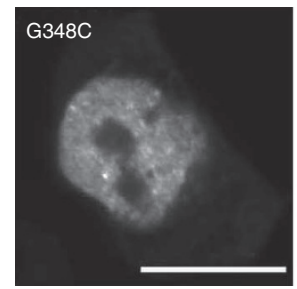

\section{R361S}

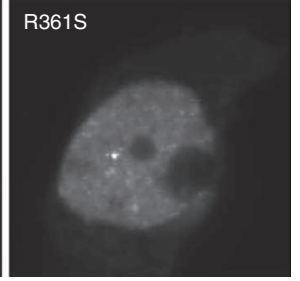

Figure 3 | A proline substitution at aggregation-prone domain of TDP-43 induces pathological degradation of TDP-43 proteins. (a) As illustrated, proline substitution could abolish the clustering of PLD if the substitute resides in a $\beta$-sheet (top); proline substitution has no effect on the clustering if the substitute resides in a loop region (bottom). (b) Immunoblot analysis of G296P, G300P, Q303P and Q343P mTDP-43 mutants. Linear illustration of the mutations substituted with proline is shown in the upper panel. Anti-GFP antibodies were used for the immunoblotting. Note that TDP-43 Q303P substitution resulted in a pathological fragment indicated by an asterisk. (c) In-vivo splicing assay of GFP-tagged mTDP-43 mutants, G296P, G300P, Q303P and Q343P ( $n=3$ ). (d) At 4 days post-transfection, cytosplasmic accumulation (arrow) and aggregation (arrowhead) was significantly increased in the TDP-43 Q303P-expressing cells. Scale bar represents $20 \mu \mathrm{m}$. (e) Quantification of cells with TDP-43 proteins accumulated in the cytosol for TDP-43-FL and TDP-43 Q303P transfectants. Here, $n=8$ for GFP-TDP-43-FL proteins and GFP-TDP-43-PLD $\Delta$ proteins; $n=12$ for GFP-TDP-43 Q303P proteins. The results of three independent experiments were analysed. (f) Comparisons of half-recovery time of fluorescence among GFP-TDP-43-FL proteins $(n=8)$, GFP-TDP-43-PLD $\Delta$ proteins $(n=8)$ and GFP-TDP-43 Q303P proteins $(n=12)$ by fluorescence recovery after photobleaching. Lines show mean + s.d. (g) MTT assay of viability of TDP-43-FL- and TDP-43-Q303P-expressing cells $(n=6)$. (h) A biochemical analysis of mTDPSupN and mTDPSup $N^{\star}$ proteins was examined using anti-GFP antibodies. The construct mTDPSupN was cleaved into an $\sim 48-\mathrm{kDa}$ fragment $\left({ }^{\star}\right)$ and a 32-kDa fragment. Tubulin and actin immunoblots were used as controls for non-specific protein degradation. (i) Localisation of GFP-tagged mTDPSupN ${ }^{\star}$. Scale bar represents $10 \mu \mathrm{m}$. (j) Deficiency of CFTR exon 9 skipping by GFP-tagged mTDPSupN ${ }^{\star}$. Exon 9 inclusion $(+)$ and exon 9 exclusion $(-)$ bands are indicated. *, aberrant splicing product $(n=3)$. ( $(\mathbf{k})$ Examination of the alternative splicing ability of ALS-linked mutants of TDP-43, G348C and R361S ( $n=3$ ). (I) Immunoblot analysis of mutation proteins. The protein lysate of G348C- and R361S-expressing cells was examined by western blot using anti-GFP antibodies. (m) Localisation of mTDP-43-G348C and mTDP-43-R361S proteins. Scale bars represent $10 \mu \mathrm{m}$. All data are presented as means with s.d.

constraining PLDs should cause oligomerlisation/functional aggregates and then impede the cleavage of TDP-43 proteins. Epigallocatechin gallate (EGCG) has been suggested to redirect an array of amyloidogenic molecules, including synuclin, huntingtin, amyloid- $\beta$, transthyretin (TTR) and yeast prion [PSI] into non-toxic oligomers. A recent study of crystallisation of a V30M TTR-EGCG complex 
further indicated that EGCG directly bound and stabilised TTR tetramer in vitro, and induced TTR oligomerisation in cells ${ }^{34}$. The pharmaceutical mechanism of EGCG curing of yeast prions [PSI] could be via stabilisation of the native prion domain Sup35 as well ${ }^{35}$. To address this, EGCG was applied to block the rapidly degraded mutant proteins of TDPSupN ${ }^{\star}$ proteins (Fig. 4a). TDPSupN ${ }^{*}$ mutants were treated with mock or $20 \mu \mathrm{M}$ EGCG. An increase in the full length TDPSupN* in cells treated with $20 \mu \mathrm{M}$ EGCG for 3 days relative to the level in mock-treated cells was observed (Fig. 4a). We further speculated that EGCG may function in preservation of the native-folded TDP-43 C terminus as well (Fig. 2). Indeed, EGCG induced oligomerisation of TDP-43 in a dosagedependent manner (Fig. 4b). In GFP-Q303P-TDP-43-expressing cells with rapid degradation, the degradation of GFP-Q303P-TDP-43 proteins was reduced by EGCG and corresponded with the appearance of TDP-43 aggregates in nuclei (Fig. 4c,d). To confirm that EGCG-induced aggregation was mediated via the TDP-43 C terminus, GFP-TDP-43-FL- or GFP-TDP-43-PLD $\Delta$-expressing cells were treated with $20 \mu \mathrm{M}$ EGCG. Upon stimulation of EGCG, GFP-TDP-43-FL proteins aggregated in the nuclei (arrow), but GFP-TDP-43-PLD $\Delta$ proteins did not (Fig. 4e). An MTT assay further confirmed that these EGCG-induced TDP-43 aggregates were non-toxic (Fig 4f). Furthermore, the normal protein degradation of GFP-TDP-43-FL proteins in confluent-transfected cells was attenuated by treating with 20 or $50 \mu \mathrm{M}$ EGCG (Fig. $4 \mathrm{~g}$ ). Consistent with the increase in TDP-43 aggregates upon EGCG treatment, the insoluble protein level of GFP-TDP-43-FL increased (Fig. 4g). The identical effect of EGCG on endogenous TDP-43 proteins was observed in confluent cells (Fig. 4h). Taken together with the results of Fig. 3, we conclude that the impairment of interactions between the cellular-folded prion domains is a prerequisite for its protein degradation under both normal and pathological conditions.

RNAs are involved in prion-like conversions of TDP-43. We searched for cellular factors affecting the prion-like conversion of TDP-43. We found a deficiency in nucleic-acid-binding-induced mTDP-43 granular assembly (Fig. 5a). GFP-tagged mTDP-43-FL and RNA-binding-deficient mutant, F147, 149L (RD) were transfected into $293 \mathrm{~T}_{\text {cells }}{ }^{36}$. Compared with cells transfected with FL, mutant transfectants showed a 20 -fold increase in granule formation (Fig. 5a). Western blotting analysis of GFP-tagged mutant F147, 149L are shown in Fig. 5b. Consistently, dimers of TDP-43 were significantly increased in the presence of RNase (Fig. 5c).

Additionally, we generated serial deletion constructs, and these are shown in Fig. 5d. The N-terminal (1-105)-deleted mTDP-43 (TDP-43-N $\Delta$ ), mTDP-43-IIPLD and mTDP-43-PLD formed large aggregates in the nucleus or cytosol that resembled misfolded inclusions, and ranged in size from 2 to $5 \mu \mathrm{m}$ and accumulated in the insoluble fraction (Fig. $5 \mathrm{~d}-\mathrm{e}$ ). The cell counts for $\mathrm{N} \Delta$-, IIPLD- and PLD-expressing transfectants significantly decreased by 67, 41 and $66 \%$, respectively (Fig. 5f). The properties of TDP-43 variants are summarised in Supplementary Table S1. Interestingly, amorphous mTDP-43-NPLD (the $\mathrm{C}$ terminus fused with the $\mathrm{N}$ terminus) proteins frequently formed doughnut-shaped aggregates, but did not cause consistent or significant cell death (Fig. 5d,f). Unlike the other a

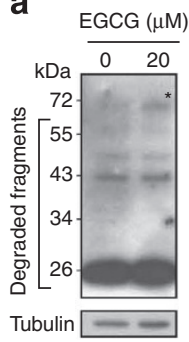

d
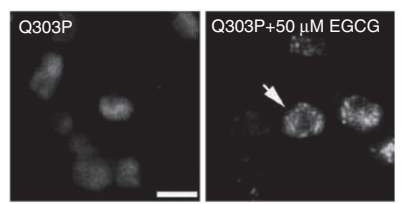

f

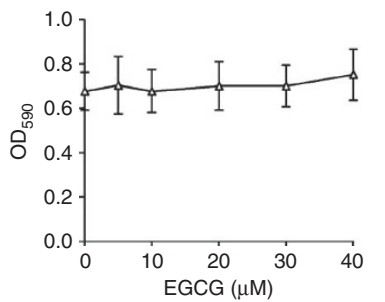

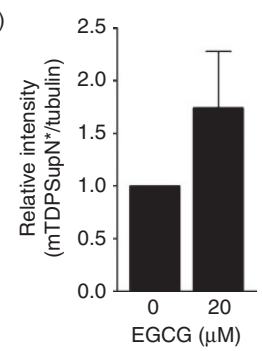

b

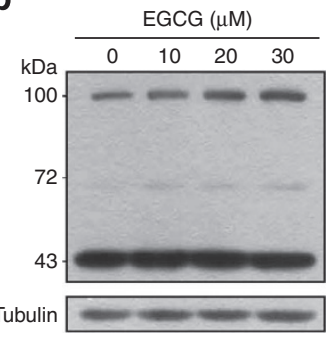

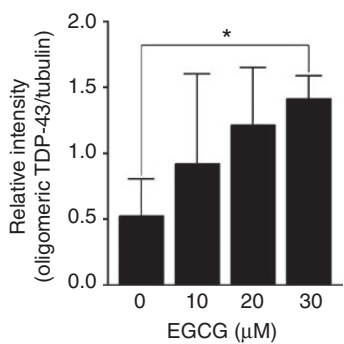

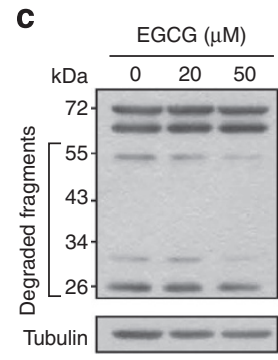

e
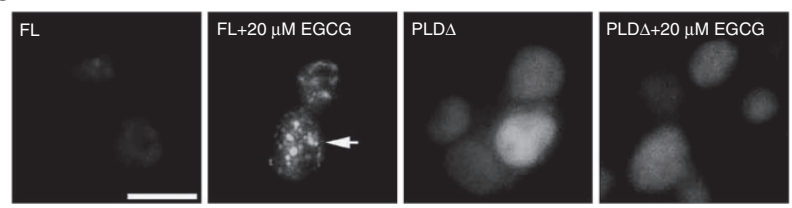

g
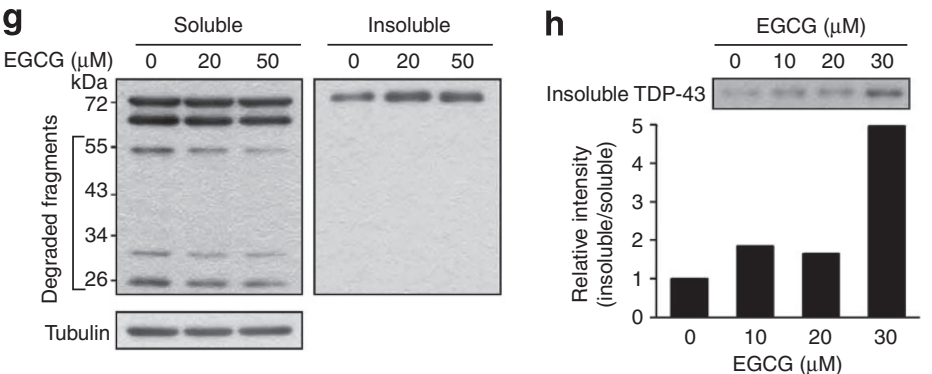

Figure 4 | The effects of EGCG on TDP-43. (a) Stimulation with $20 \mu$ M EGCG increased the FL of TDPSupN* ${ }^{\star}$. The TDPSupN-expressing cells were treated with or without EGCG for 3 days and were then harvested for analysis using anti-GFP antibodies. ${ }^{*} F L(n=3)$. (b) A biochemical analysis of endogenous proteins with or without EGCG using anti-TDP-43 antibodies $(n=3)$. ${ }^{\star} P<0.05$ by analysis of variance. (c) EGCG attenuated the protein degradation of GFP-Q303P-TDP-43. (d) Upon EGCG addition, GFP-Q303P-TDP-43 aggregates (arrow) appeared by using anti-GFP antibodies. Scale bar represents $10 \mu \mathrm{m}$. (e) The treatment of PLD truncated mTDP-43 mutant with EGCG. Scale bar represents $10 \mu \mathrm{m}$. (f) MTT assay of viability of TDP-43-FL-expressing cells given EGCG $(n=5)$. (g) A biochemical analysis of GFP-TDP-43-FL proteins with or without EGCG using anti-GFP antibodies. (h) A biochemical analysis of endogenous proteins with or without EGCG using anti-TDP-43 antibodies. Data are presented as means $(n=2)$. 
a

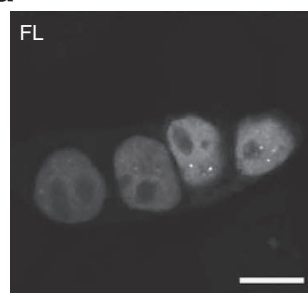

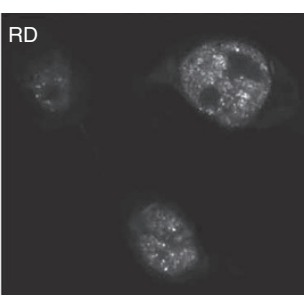
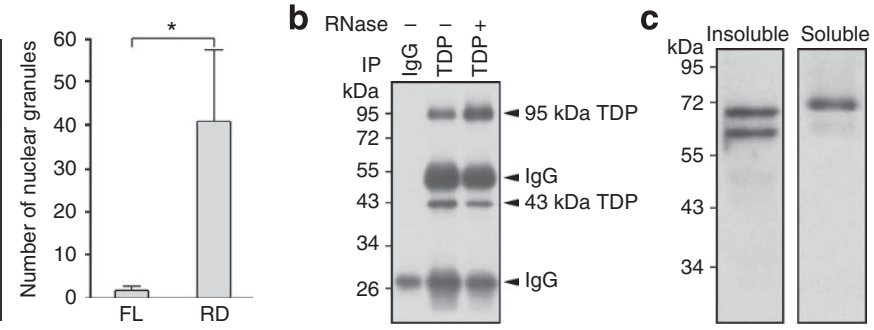

d
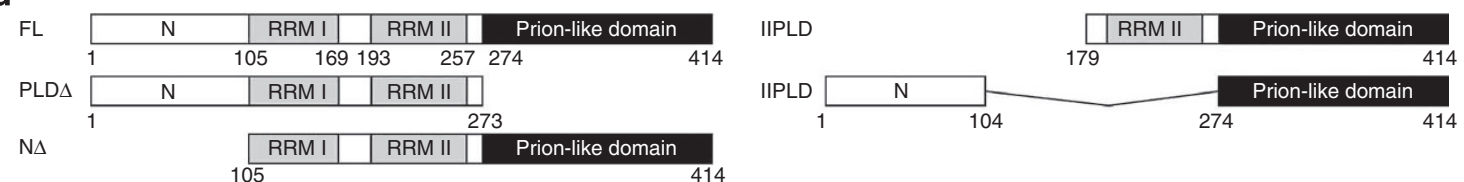

105
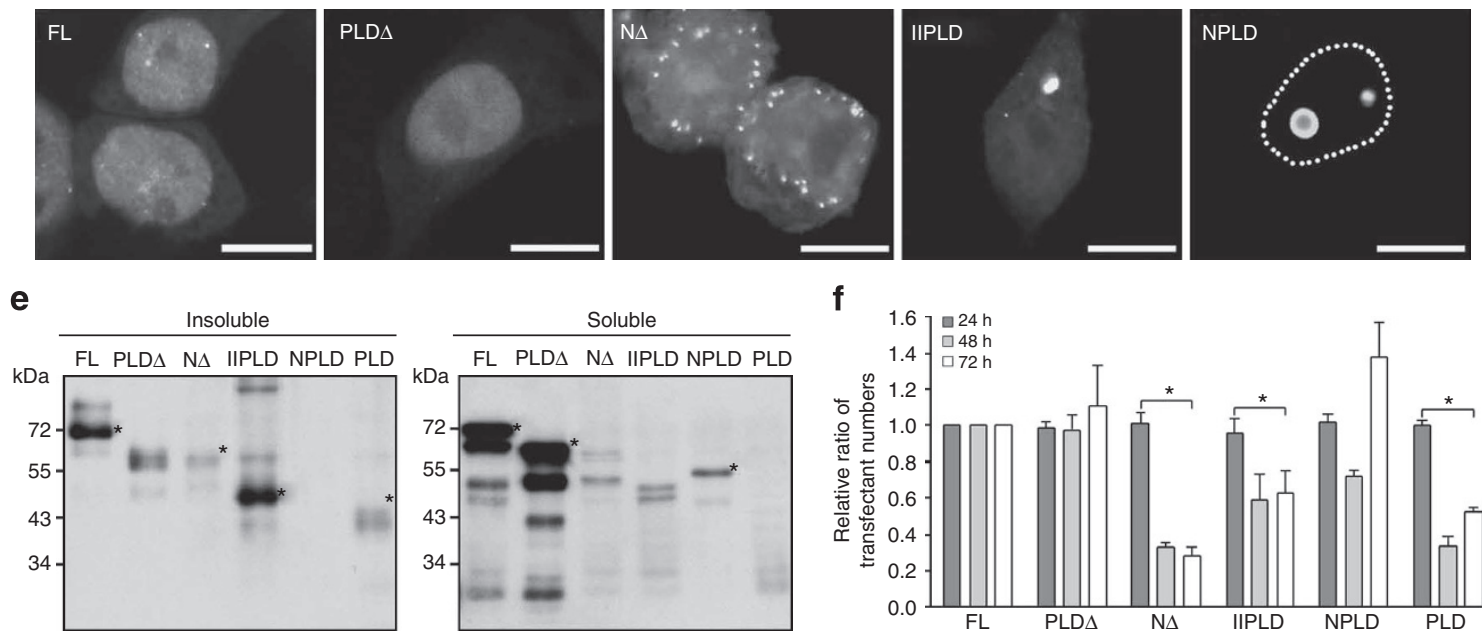

$\mathbf{f}$

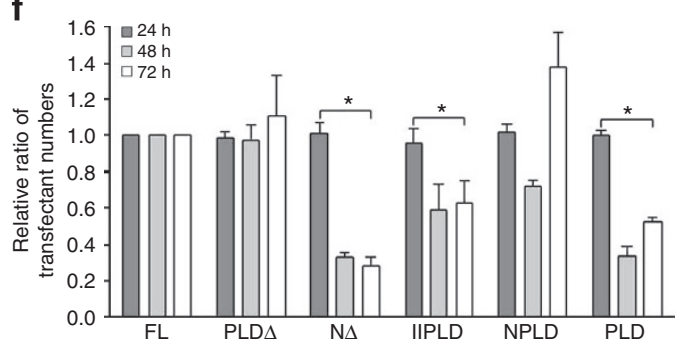

Figure 5 | The effects of RNAs or TDP-43 N terminus on intrinsic propensity of TDP-43 C terminus. (a) GFP-tagged mTDP-43 FL and the mutant F147, 149L (RD) were transfected into 293T cells. Compared with cells transfected with FL, mutant transfectants showed a 20-fold increase in granule formation. The results of three independent experiments were analysed. All data are presented as means with s.d. $(n=3)$. ${ }^{*} P<0.05$ by Student's t-test. (b) An examination of TDP-43 proteins in presence of RNase. The protein lysate of 293T cells was used for immunoprecipitation with anti-TDP-43 antibody and was further examined by immunoblotting with anti-TDP antibody. Dimers of TDP were increased in presence of RNase. (c) Western blotting analysis of the solubility of GFP-tagged mutant F147, 149L. (d) The truncated mTDP-43 variants containing PLD formed aggregates in 293 T cells. A scheme of the TDP-43 construct, top, illustrates functional domains. N $\Delta$, IIPLD and NPLD, but not PLD $\Delta$, formed aggregates in the nucleus or cytosol. Amorphous mTDP-43-NPLD (the $C$ terminus fused with the $N$ terminus) proteins frequently formed doughnut-shaped aggregates. Scale bars represent $10 \mu \mathrm{m}$. (e) Validation of the solubility of GFP-tagged mTDP-43 variants by western blot; ${ }^{\star}$, expected molecular weight. (f) Examination of the toxicity of TDP-43 aggregates. The 293T cells were transfected with GFP-tagged variants of mTDP-43. GFP-positive cells were counted at 24,48 and $72 \mathrm{~h}$ post-transfection. All data are presented as means with s.d. $(n=5)$. ${ }^{\star} P<0.05$ by analysis of variance.

proteins containing PLD, mTDP-43-NPLD remained in soluble fraction, suggesting possible modification of solubility of the PLD by the $\mathrm{N}$ terminus in cells (Fig. $5 \mathrm{e}$ ).

\section{Discussion}

Increasing evidence indicates that aggregation-prone domains have crucial roles in various cellular functions, such as granular assembly and nuclear export (Table 1). In this study, we further found that the Q/N-driving propensity of TDP-43 is involved in alternative splicing and protein stability. Recently, an increasing number of prionogenic domain-containing proteins have been identified; among them, many, such as Mot3p and Lsm4p, contain a Q/N-rich domain and are involved in transcription or RNA processing ${ }^{37}$. Additionally, a self-perpetuating Q/N-rich domain of apCPEB was suggested to participate in the long-term facilitation of Aplysia ${ }^{31}$. A set of proteins that control the stability of mRNAs as well as their translational silencing are colocalised in the cytoplasmic foci called processing bodies or P-bodies ${ }^{38}$. Interestingly, several P-body components contain a potential prionogenic domain. In yeast, the prion-like properties of this motif may be related to granule assembly and can be restored by replacement with yeast prion RNQ1. Lsm $4 p$ is one of these well-characterised proteins ${ }^{27}$. Ultrastructural studies have demonstrated that the P-bodies are condensed fibrillar aggregates as observed in the yeast prion [PSI $]^{39,40}$. In the same way as shown here in the assembly of TDP-43 functional aggregates, assembly of P-bodies is dynamic and, in neurons, is regulated by the neuronal activity ${ }^{41}$. These observations are reminiscent of the non-toxic $\sim 500 \mathrm{kDa}$ oligomers of amyloidogenic molecules found in a new type of pathogenesis pathway ${ }^{42}$. We suggested that the higher bands of TDP-43 shown in Fig. 2i-k, would be assembled prion-like oligomers of TDP-43, as the overexpression of TDP-43 Q303P interfered with the generation of endogenous higher bands of TDP-43 upon oxidative stress (Chang, H. Y. and Wang, I. F., unpublished data). 


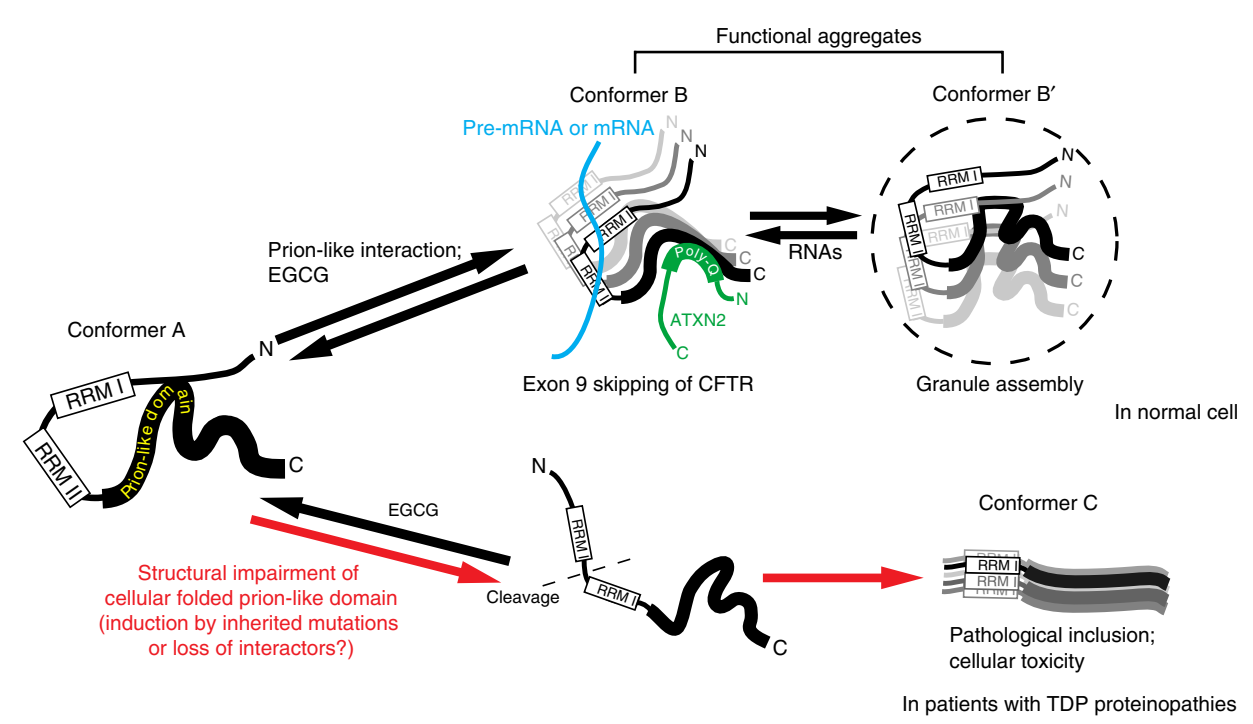

Figure 6 | Working model for the intrinsic propensities of TDP-43 GQN-rich C terminus in normal cellular functions and disease progression.

As part of the normal physiological processes of the cell, the intrinsic propensity of the TDP-43 C terminus is to confer assembly of nuclear bodies, protein stability, and promote exon 9 skipping of CFTR by adopting flexible prion-like folding; however, if loss of TDP-43 N terminus by pathological cleavage, ALS-linked mutations or other unknown cellular factors could cause disruption of cellular prion-like folding of the TDP-43 C terminus, TDP-43 inclusions will form. EGCG induced oligomerisation of TDP-43 and reduced its degradation.

On the basis of the results of the current study, we propose a model, outlined in Fig. 6, in which the ambivalent propensity of the TDP-43 C terminus forms either structurally flexible aggregates under physiological processes (Fig. 6, Conformer B and Conformer $\mathrm{B}^{\prime}$ ), or rigid pathological inclusions (Fig. 6, Conformer C). The restorative formation of nuclear clustering and alternative splicing ability when TDP-43 C terminus is replaced by yeast Sup35N strongly suggests that a prion-like property is involved in subcellular localisation and exon 9 skipping of CFTR (Fig. 2). Interestingly, ALS-inherited G348C and R361S mutants attenuated exon 9 skipping efficiency by 12.5 and $28.9 \%$, respectively, whereas G296P, a disease-unrelated mutant, did not (Fig $3 \mathrm{k}-\mathrm{m})$. These results implied that ALS-inherited mutations showed attenuation of the prion-like activity of TDP-43. We propose that the impairment of interactions between PLDs occurs before caspase cleavage, as an oligomer stabiliser, EGCG, can reduce protein degradation of TDP-43 mutants defective in prion-like interaction (Fig. 4a,c,d). The failure of the PLD to adopt proper cellular folding due to a loss of prion-like interactions or impairment by ALS-linked mutations at the early stages of pathogenesis may trigger pathological fragment cleavage, convert the PLD to a misfolded structure and ultimately result in disease inclusions. We also found that the TDP- $43 \mathrm{~N}$ terminus stabilised the cellular-folded conformation and altered the insoluble propensity of TDP-43 C terminus, suggesting that the TDP-43 $\mathrm{N}$ terminus (1-105) may act as a chaperon-like domain to autoinhibit the disorder tendency of the PLD through a long-range intramolecular interaction (Fig. 5d-f). A recent study showed that physical interaction of mutant SOD1 proteins with the TDP-43 $\mathrm{N}$ terminus consistently resulted in an increase in TDP-43 proteins in the detergent-insoluble fraction ${ }^{43}$.

Additionally, the coimmunoprecipitation of endogenous TDP with mTDPSupN suggested that prion-like properties mediate the interactions between heterogeneous Q/N-rich domains (Fig. 2). Indeed, we have found that proteins containing polyQ domains are often functionally linked to other poly-(amino acid) proteins by database analysis (http://string.embl.de/) of the functional interactomes of $\mathrm{Q} / \mathrm{N}$-rich proteins. For example, functional interactome analysis of ATXN2 reveals ten functional interactors. Eight of the ten interactors, FOX1, TBP, ATN1, CACNA1A, ATXN1, ATXN3, ATXN7 and DDX6 contain a polyQ-rich element. In addition to the mTDPSupN used in this study, TDP-43 has recently been shown to interact with DDX6 and ATXN2 ${ }^{44-46}$. These three Q/N-rich proteins could physically interact with each other and localise to stress granules or P-bodies under certain conditions ${ }^{45}$. More recently, Elden et al. ${ }^{44}$ demonstrated that treatment with RNase abolished coimmunoprecipitatation of ATXN2 with TDP-43-YFP, suggesting that the interaction of ATXN2 and TDP-43 occurs in an RNA-dependent manner. With regard to the RNA-dependent regulation of TDP-43 activity, we found that RNA-bindingdeficient mutants of TDP-43 proteins would form visible granular aggregates and gained an $\sim 20$-fold TDP-43 visible granules in the nucleus (Fig. 5a) ${ }^{44}$. The relocalisation of TDP43 proteins from irregular puncta to visible granules, where TDP-43 did not bind to RNAs, suggests that prion-like activity may be regulated by RNA binding. We propose that the Q/N-rich element of TDP-43 can be exposed when RNAs bind to TDP-43. The allosteric binding can allow natively folded TDP-43 to interact with natively folded ATXN2 or DDX6 in a prion-like manner (Fig. 6, Conformer B). In the presence of RNase, a significant shifting of TDP-43 from monomer to dimer was observed by western blotting analysis (Fig. 5c). Together with observations in Fig. $5 \mathrm{a}-\mathrm{b}$, and these results supported in the absence of RNA, TDP-43 proteins formed the other type of functional aggregates, proposed as Conformer B' of Fig. 6.

The cross-interactions between 'cellular-folded Q/N-rich domains' provide an explanation for the reciprocal effects of prone-to-aggregation protein in common protein-misfolding disease. The biochemical and biophysical roles of the cellular-folded $\mathrm{Q} / \mathrm{N}$-rich domain are likely to retain the protein in the insoluble fraction by self- or cross-interaction (Fig. 2 and Table 1). This function would allow the protein to form dynamic scaffolds in a stable conformation and have a lower mobility, which is suited for restricting the protein to a microenvironment optimal for performing particular 
bioreactions, such as alternative splicing and sub-compartment assembly.

As the aggregation-prone domain has structural plasticity and can spontaneously self-assemble into ordered pathological or functional aggregates, it is reasonable to speculate that these two pathways may act antagonistically, as shown in Fig. 6. Ideally, compounds that stabilise functional aggregates of aggregation-prone domains may be investigated as therapies for protein misfolding. This is particularly important for TDP-43 proteinopathies, because cleavage of TDP-43 proteins occurs before inclusion formation during disease progression. Loss of normal TDP-43FL proteins perturbs pre-mRNA splicing and ultimately leads to cell death. Growing evidence shows that polyphenol compounds, such as EGCG, redirect an array of amyloidogenic molecules and effectively cured huntingtin inclusions by oral administration in a Drosophlia melanogaster model ${ }^{47,48}$. In our studies, EGCG induced oligomerlisation of natively folded TDP-43 and thus inhibited pathological-like degradation, suggesting targeting to stabilisation of natively folded prion domains is a promised therapeutic approach for the frontotemporal lobar degeneration with ubiquitin-positive inclusions and ALS. With an eye to therapy design for command of cellular-folded aggregation-prone domain, future work should focus on the resolution of an accurate cellular structure of the TDP-43 C terminus and the identification of factors affecting prion-like interactions under physiological and pathological processes. A potential mechanism could be the interplay of post-translation modifications on the PLD.

Herein, we found that in normal cells, the cellular-folded TDP$43 \mathrm{C}$ terminus is prone to aggregation and tends to retain TDP43 proteins in the insoluble form, as does the misfolded protein. In contrast to the misfolded protein, however, the cellular TDP-43 aggregates assembled highly dynamically and were regulated by stimuli. Substitutions of the C terminus of mammalian TDP-43 with a yeast prion domain, Sup35N, recapitulated granular assembly and dynamics, effective CFTR exon-9 skipping, intrinsic element- and self-interactions, and insolubility. Impairing prion-like interactions of TDP-43 caused its degradation into a fragment identified in patients with early-stage TDP-43 proteinopathies. We conclude that the role of natively folded TDP-43 C terminus is directly associated with a loss-of-function in TDP-43 proteinopathies at early pathogenesis. Additionally, a useful approach to specifically interfere with prion-like interactions in vivo by proline substitution was developed in this study, and could be widely applied to determine cellular roles of other Q/N-rich proteins.

\section{Methods}

Plasmids. All primers used for plasmid construction are listed in Supplementary Table S2. The FL and truncated mTDP-43 cDNAs were amplified from mouse cDNA using primers containing HindIII and XmaI sites, and subsequently ligated into pEGFP-N3 (Clontech) to obtain GFP-tagged TDP-43 variants. SupN and SupN ${ }^{*}$ cDNAs were individually amplified using YCp-I-SupF and YCp-I-SupF(G25P) as templates, respectively, and ligated into XmaI digestedmTDPPLD $\Delta$. For construction of mTDPNPLD and MTDPRD, mega-primers were initially amplified by primer 1 and primer 2 from mouse cDNA. The resulting mega-primers were used to generate MTDPNPLD and mTDPRD fragments from mouse $\mathrm{cDNA}$ with primer 3 . The mTDPNPLD and mTDPRD $\mathrm{cDNAs}$ were cloned into pEGFP-N3 (Clontech) following HindIII and XmaI digestion. G296P, G348C and R361S were generated by Pfu Turbo (Stratagene).

Living cell imaging. A total of 293 cells were grown on cover slides and then transfected with GFP-tagged plasmids. All 16-bit images were obtained by ZEISS LSM 710 and a 63/1.4 NA lens was used for these experiments. Cells were always kept at $37^{\circ} \mathrm{C}, 5 \% \mathrm{CO}_{2}$. For fluorescence recovery after photobleaching, the laser-scanning microscope was set to laser spectrum at a wavelength of $488 \mathrm{~nm}$ and the intensity of $<1.25 \%$ of its total power (25\%). A nuclear region of interest in the transfectant was defined and then photobleached, using 100 iterations at $100 \%$ laser intensity. After $10 \mathrm{~s}$ recording, the cells were photobleached, following by $1 \mathrm{~s}$-interval recording. As there were no significant changes in GFP signal equilibrium after $180 \mathrm{~s}$, we set the total recording time to $200 \mathrm{~s}$.
In-vivo splicing assay. The splicing assay was performed as previously described by Buratti et al ${ }^{10}$. In brief, $3 \mu \mathrm{g}$ of hCF-TG13T5 minigene was cotransfected with $3 \mu \mathrm{g}$ of GFP-tagged mTDP-43 variants into 293T cells. Forty-eight hours after transfection, total RNA was isolated by TRIzol (Invitrogen). RT-PCR was performed using $5 \mu \mathrm{g}$ of total RNA and SuperScript III reverse transcriptase (Invitrogen). The resulting cDNA was then submitted to amplification of the splicing products with the specific primers Bra2 (5'-TAGGATCCGGTCACCAG GAAGTTGGTTAAATCA-3') and a2-3 (5'-CAACTTCAAGCTCCTAAGCCACT GC- $\left.3^{\prime}\right)$. For statistical analysis, three experiments were performed, and the resulting images were quantified by AlphaEaseFC Version 3.2.1 (Alpha Innotech).

Solubility assay of GFP-tagged mTDP-43 variants. GFP-tagged mTDP- $43(3 \mu \mathrm{g})$ variant expression plasmid was transfected into 293T cells. Forty-eight hours after transfection, cells were lysed by RIPA $(50 \mathrm{mM}$ Tris, $150 \mathrm{mM} \mathrm{NaCl}, 1 \mathrm{mM}$ EDTA, $1 \% \mathrm{NP}-40, \mathrm{pH} 7.4)$ and fractionated by centrifugation at $16,000 \mathrm{~g}$ for $15 \mathrm{~min}$ at $4{ }^{\circ} \mathrm{C}$. The pellet was then dissolved in $8 \mathrm{M}$ urea $/ 50 \mathrm{mM}$ Tris, pH 8.0. GFP-tagged mTDP-43 variants were identified by western blot using a monoclonal GFP antibody (632,381, BD Biosciences Clontech).

Cell culture and transfection. The $293 \mathrm{~T}$ cells were grown in Dulbecco's modified Eagle's medium, supplemented with $10 \%$ fetal bovine serum, $1 \%$ penicillin/ streptomycin and $1 \% \mathrm{~L}$-glutamate. Primary cultures of rat hippocampal neurons were prepared from embryonic day 17 rat embryos as previously described by Wang et $a l^{7}$. All procedures for handling the rat were carried out in accordance with the guidelines approved by the Institutional Animal Care and Utilization Committee, Academia Sinica. Hippocampal cells were plated on poly-l-lysinecoated coverslides at low density $\left(10,000\right.$ cells per $\left.\mathrm{cm}^{2}\right)$ and cultured in the neurobasal medium/B27 (Invitrogen). The 293T cells were transfected using the calcium phosphate protocol according to the manufacturer's instructions. Primary neurons were transfected using Lipofectamine 2000 (Invitrogen) at DIV3. The luciferase activity following cotransfection with the reporter plasmid, pG0- $\alpha$-Luc, and GFP-tagged mTDP-43 variants were assayed following the manufacturer protocol (Promega). The luciferase activity was further calibrated by cell numbers ${ }^{12}$

Image acquisition. The cells were fixed with $3.7 \%$ formaldehyde in PBS at $4{ }^{\circ} \mathrm{C}$ for $15 \mathrm{~min}$. Cover slips were mounted with mounting medium, and the fluorescent images were analysed using a laser confocal microscope LSM510 META (Zeiss). Each confocal image was obtained from a single optical section.

MTT assay. The $293 \mathrm{~T}$ cells $\left(1 \times 10^{5}\right)$ were seeded in each well of a 24 -well plate, and incubated overnight in serum containing Dulbecco's modified Eagle's medium/F12 in a $37^{\circ} \mathrm{C}$ incubator with $5 \% \mathrm{CO}_{2}$. Cells were then transfected with GFP-tagged mTDP-43 variants. After $12 \mathrm{~h}$ post-transfection, cells were treated with EGCG (10, $20,30,40,50 \mu \mathrm{M})$ for $24 \mathrm{~h}$. MTT assay was performed following the user manual (Promega). The optical absorbance was measured at $590 \mathrm{~nm}$, and five independent experiments were performed.

Electronic microscopy study. The labelling procedure followed that described by Liou et al. ${ }^{48}$, with some modification. Briefly, the 293T cells transfected with GFPtagged PLD were first fixed by incubation in cold $0.1 \mathrm{M}$ phosphate-citrate buffer (pH 7.2) containing $1 \%$ glutaraldehyde and $1 \%$ formaldehyde (Merck) at $4{ }^{\circ} \mathrm{C}$ overnight. The 293T cells were then neutralised with $0.1 \mathrm{M}$ ammonium chloride and embedded with LR-Gold reagent. The ultrathin sections of embedded samples were stained with uranyl acetate and lead citrate. Samples were examined using a Philip Tecnai T12 electron microscope.

Statistical analyses. The statistical significance was evaluated by analysis of variance. The difference between groups was considered as significant when a $P$-value was $<0.05$

\section{References}

1. Neumann, M. et al. Ubiquitinated TDP-43 in frontotemporal lobar degeneration and amyotrophic lateral sclerosis. Science 314, 130-133 (2006).

2. Neumann, M., Kwong, L. K., Sampathu, D. M., Trojanowski, J. Q., \& Lee, V. M. TDP-43 proteinopathy in frontotemporal lobar degeneration and amyotrophic lateral sclerosis: protein misfolding diseases without amyloidosis. Arch. Neurol. 64, 1388-1394 (2007).

3. Kabashi, E. et al. TARDBP mutations in individuals with sporadic and familial amyotrophic lateral sclerosis. Nat. Genet. 40, 572-574 (2008).

4. Winton, M. J. et al. Disturbance of nuclear and cytoplasmic TAR DNA-binding protein (TDP-43) induces disease-like redistribution, sequestration, and aggregate formation. J. Biol. Chem. 283, 13302-13309 (2008).

5. Lin, W. L. \& Dickson, D. W. Ultrastructural localization of TDP-43 in filamentous neuronal inclusions in various neurodegenerative diseases. Acta Neuropathol. 116, 205-213 (2008).

6. Hasegawa, M. et al. Phosphorylated TDP-43 in frontotemporal lobar degeneration and amyotrophic lateral sclerosis. Ann. Neurol. 264, 60-70 (2008). 
7. Wang, I. F., Wu, L. S., Change, H. Y. \& Shen, C. K. TDP-43, the signature protein of FTLD-U, is a neuronal activity-responsive factor. J. Neurochem. 105, 797-806 (2008).

8. Zhang, Y. J. et al. Aberrant cleavage of TDP-43 enhances aggregation and cellular toxicity. Proc. Natl Acad. Sci. USA 106, 7607-7612 (2009).

9. Wang, H. Y., Wang, I. F., Bose, J. \& Shen, C. K. Structural diversity and functional implications of the eukaryotic TDP gene family. Genomics 83, 130-139 (2004).

10. Ou, S. H., Wu, F., Harrich, D., Garcia-Martinez, L. F. \& Gaynor, R. B. Cloning and characterization of a novel cellular protein, TDP-43, that binds to human immunodeficiency virus type 1 TAR DNA sequence motifs. J. Virol. 69, 3584-3596 (1995)

11. Buratti, E. et al. Nuclear factor TDP-43 and SR proteins promote in vitro and in vivo CFTR exon 9 skipping. EMBO J. 20, 1774-1784 (2001).

12. Wang, I. F., Reddy, N. M. \& Shen, C. K. Higher order arrangement of the eukaryotic nuclear bodies. Proc. Natl Acad. Sci. USA 99, 13583-13588 (2002).

13. Tollervey, J. R. et al. Characterizing the RNA targets and position-dependent splicing regulation by TDP-43. Nat. Neurosci. 14, 452-458 (2011).

14. Buratti, E. et al. TDP-43 binds heterogeneous nuclear ribonucleoprotein A/B through its C-terminal tail: an important region for the inhibition of cystic fibrosis transmembrane conductance regulator exon 9 splicing. J. Biol. Chem. 280, 37572-37584 (2005).

15. Wang, I. F., Wu, L. S. \& Shen, C. K. TDP-43: an emerging new player in neurodegenerative diseases. Trends Mol. Med. 14, 479-485 (2008).

16. Fuentealba, R. A. et al. Interaction with polyglutamine aggregates reveals a Q/N-rich domain in TDP-43. J. Biol. Chem. 285, 26304-26314 (2010).

17. Johnson, B. S. et al. TDP-43 is intrinsically aggregation-prone, and amyotrophic lateral sclerosis-linked mutations accelerate aggregation and increase toxicity. J. Biol. Chem. 284, 20329-20339 (2009).

18. Bregman, D. B., Du, L., van der Zee, S. \& Warren, S. L. Transcriptiondependent redistribution of the large subunit of RNA polymerase II to discrete nuclear domains. J. Cell Biol. 129, 287-298 (1995).

19. Johnson, B. S., McCaffery, J. M., Lindquist, S. \& Gitler, A. D. A yeast TDP-43 proteinopathy model: exploring the molecular determinants of TDP-43 aggregation and cellular toxicity. Proc. Natl Acad. Sci. USA 105, 6439-6444 (2008)

20. Brandner, S. et al. Normal host prion protein necessary for scrapie-induced neurotoxicity. Nature 379, 339-343 (1996).

21. Paushkin, S. V., Kushnirov, V. V., Smirnov, V. N. \& Ter-Avanesyan, M. D. Propagation of the yeast prion-like [psi+] determinant is mediated by oligomerization of the SUP35-encoded polypeptide chain release factor. EMBO J. 15, 3127-3134 (1996).

22. Patino, M. M., Liu, J. J., Glover, J. R. \& Lindquist, S. Support for the prion hypothesis for inheritance of a phenotypic trait in yeast. Science 273, 622-626 (1996).

23. True, H. L. \& Lindquist, S. L. A yeast prion provides a mechanism for genetic variation and phenotypic diversity. Nature 407, 477-483 (2000).

24. Derkatch, I. L. et al. Effects of Q/N-rich, polyQ, and non-polyQ amyloids on the de novo formation of the [PSI+] prion in yeast and aggregation of Sup35 in vitro. Proc. Natl Acad. Sci. USA 101, 12934-12939 (2004).

25. Gilks, N. et al. Stress granule assembly is mediated by prion-like aggregation of TIA-1. Mol. Biol. Cell 15, 5383-5398 (2004).

26. Tanaka, M., Chien, P., Naber, N., Cooke, R. \& Weissman, J. S. Conformational variations in an infectious protein determine prion strain differences. Nature 428, 323-328 (2004)

27. Decker, C. J., Teixeira, D. \& Parker, R. Edc3p and a glutamine/asparagine-rich domain of Lsm $4 p$ function in processing body assembly in Saccharomyces cerevisiae. J. Cell Biol. 179, 437-449 (2007).

28. Vitrenko, Y. A., Gracheva, E. O., Richmond, J. E. \& Liebman, Z. S. W. Visualization of aggregation of the Rnq1 prion domain and cross-seeding interactions with Sup35 NM. J. Biol. Chem. 282, 1779-1787 (2007).

29. Wickner, R. B., Shewmaker, F., Kryndushkin, D. \& Edskes, H. K. Protein inheritance (prions) based on parallel in-register beta-sheet amyloid structures. Bioessays 30, 955-964 (2008).

30. Ader, C. et al. Amyloid-like interactions within nucleoporin FG hydrogels. Proc. Natl Acad. Sci. USA 107, 6281-6285 (2010).

31. Si, K., Choi, Y. B., White-Grindley, E., Majumdar, A. \& Kandel, E. R. Aplysia $\mathrm{CPEB}$ can form prion-like multimers in sensory neurons that contribute to long-term facilitation. Cell 140, 421-435 (2010).

32. Soto, C., Kindy, M. S., Baumann, M. \& Frangione, B. Inhibition of Alzheimer's amyloidosis by peptides that prevent beta-sheet conformation. Biochem. Biophys. Res. Commun. 226, 672-680 (1996).

33. Chang, H. Y., Lin, J. Y., Lee, H. C., Wang, H. L. \& King, C. Y. Strain-specific sequences required for yeast [PSI+] prion propagation. Proc. Natl Acad. Sci. USA 105, 13345-13350 (2008).
34. Miyata, M. et al. The crystal structure of the green tea polyphenol (-)-epigallocatechin gallate-transthyretin complex reveals a novel binding site distinct from the thyroxine binding site. Biochemistry 49, 6104-6114 (2010).

35. Roberts, B. E. et al. A synergistic small-molecule combination directly eradicates diverse prion strain structures. Nat. Chem. Biol. 5, 936-946 (2009).

36. Buratti, E. \& Baralle, F. E. Characterization and functional implications of the RNA binding properties of nuclear factor TDP-43, a novel splicing regulator of CFTR exon 9. J. Biol. Chem. 276, 36337-36343 (2001).

37. Alberti, S., Halfmann, R., King, O., Kapila, A. \& Lindquist, S. A. A systematic survey identifies prions and illuminates sequence features of prionogenic proteins. Cell 137, 146-158 (2009).

38. Fenger-Grøn, M., Fillman, C., Norrild, B. \& Lykke-Andersen, J. Multiple processing body factors and the ARE binding protein TTP activate mRNA decapping. Mol. Cell 20, 905-915 (2005).

39. Souquere, S. et al. Unravelling the ultrastructure of stress granules and associated P-bodies in human cells. J. Cell Sci. 122, 3619-3626 (2009).

40. Kawai-Noma, S. et al. In vivo evidence for the fibrillar structures of Sup35 prions in yeast cells. J. Cell Biol. 190, 223-231 (2010).

41. Zeitelhofer, M. et al. Dynamic interaction between P-bodies and transport ribonucleoprotein particles in dendrites of mature hippocampal neurons. J. Neurosci. 28, 7555-7562 (2008).

42. Behrends, C. et al. Chaperonin TRiC promotes the assembly of polyQ expansion proteins into nontoxic oligomers. Mol. Cell 23, 887-897 (2006).

43. Yoon, E. J. et al. Intracellular amyloid beta interacts with SOD1 and impairs the enzymatic activity of SOD1: implications for the pathogenesis of amyotrophic lateral sclerosis. Exp. Mol. Med. 41, 611-617 (2009).

44. Elden, A. C. et al. Ataxin-2 intermediate-length polyglutamine expansions are associated with increased risk for ALS. Nature 466, 1069-1075 (2010).

45. Nonhoff, U. et al. Ataxin-2 interacts with the DEAD/H-box RNA helicase DDX6 and interferes with P-bodies and stress granules. Mol. Biol. Cell 18, 1385-1396 (2007).

46. Freibaum, B. D., Chitta, R. K., High, A. A. \& Taylor, J. P. Global analysis of TDP-43 interacting proteins reveals strong association with RNA splicing and translation machinery. J. Proteome Res. 9, 1104-1120 (2010).

47. Bieschke, J. et al. EGCG remodels mature alpha-synuclein and amyloid-beta fibrils and reduces cellular toxicity. Proc. Natl Acad. Sci. USA 107, 7710-7715 (2010).

48. Ehrnhoefer, D. E. et al. Green tea (-)-epigallocatechin-gallate modulates early events in huntingtin misfolding and reduces toxicity in Huntington's disease models. Hum. Mol. Genet. 15, 2743-2751 (2006).

49. Liou, G. G., Jane, W. N., Cohen, S. N., , Lin, N. S. \& Lin-Chao, N. S. RNA degradosomes exist in vivo in Escherichia coli as multicomponent complexes associated with the cytoplasmic membrane via the $\mathrm{N}$-terminal region of ribonuclease E. Proc. Natl Acad. Sci. USA 98, 63-68 (2001).

\section{Acknowledgements}

We are grateful to Dr Chi-Huey Wong at Academia Sinica for discussion and proofreading, to Tsung-Yu Tsai for her assistance in writing this article, and to Dr F Baralle at the International Centre for Genetic Engineering and Biotechnology in Italy for providing us with the hCF-TG13T5 minigene plasmid. We also thank the staff of the Imaging Core of IMB, Academia Sinica. This research was supported by Academia Sinica, National Health Research Institutes and National Science Council grants to C.-K. J.S. (NSC 99-2321-B-001-033) and G.-G.L. (MG-099-PP-08), and Garage Brain Science Com. (ND-01-001). I.-F.W. dedicates this article to her late sister.

\section{Author contributions}

I.-F.W. conceived the research, designed and performed the experiments, wrote the manuscript. H.-Y.C. performed bioinformatic analysis, conducted the experiments and assisted in writing the manuscript. S.-C.H. and T.-D.W. assisted in EGCGrelated experiments. G.-G.L. carried out the EM. C.-K.J. S. helped in revision of the manuscript.

\section{Additional information}

Supplementary Information accompanies this paper at http://www.nature.com/ naturecommunications

Competing financial interests: The authors declare no competing financial interests.

Reprints and permission information is available online at http://npg.nature.com/ reprintsandpermissions/

How to cite this article: Wang, I.-F. et al. The self-interaction of native TDP-43 $\mathrm{C}$ terminus inhibits its degradation and contributes to early proteinopathies. Nat. Commun. 3:766 doi: 10.1038/ncomms1766 (2012). 